\title{
Activation of rat liver microsomal glutathione transferase by limited proteolysis
}

\author{
Ralf MORGENSTERN, ${ }^{*} \S$ Gerd LUNDQUIST, ${ }^{*}$ Hans JÖRNVALL $\dagger$ and Joseph W. DePIERRE $\ddagger$ \\ *Department of Toxicology, Karolinska Institutet, S-104 01 Stockholm, †Department of Chemistry I, Karolinska Institutet, \\ S-104 01 Stockholm, and ‡Department of Biochemistry, Arrhenius Laboratory, University of Stockholm, \\ S-106 91 Stockholm, Sweden
}

\begin{abstract}
The activity of rat liver microsomal glutathione transferase is increased by limited tryptic proteolysis; the membrane-bound and purified forms of the enzyme are activated about 5- and 10-fold respectively. The cleavage sites that correlate with this activation were determined by amino acid sequence analysis to be located after Lys-4 and Lys-41. Differences in the relative extent of cleavage at these two sites did not consistently affect the degree of activation. Thus the data support the conclusion that cleavage at either site results in activation. The trypsin-activated enzyme was compared with the form activated with $N$ ethylmaleimide, which modifies Cys-49. These two differently activated forms were found to have similar kinetic parameters, which differ from those of the unactivated enzyme. The relatedness of the two types of activation is also demonstrated by the observation that microsomal glutathione transferase fully activated by $N$-ethylmaleimide is virtually resistant to further activation by trypsin. This is the case despite the fact that the $N$-ethylmaleimide-activated enzyme is much more susceptible to trypsin cleavage at Lys- 41 than is the untreated enzyme. The latter observation indicates that activation with $N$-ethylmaleimide is accompanied by a conformational change involving Lys- 41 .
\end{abstract}

\section{INTRODUCTION}

Glutathione transferases are a group of enzymes that conjugate numerous carcinogenic, mutagenic, toxic and pharmacologically active compounds with glutathione [1]. There are at least ten cytosolic forms of this enzyme, all with broad and overlapping substrate specificity [2], and at least one microsomal form (see [3] for a review).

We have studied the membrane-bound microsomal glutathione transferase with regard to substrate specificity, amino acid sequence and oligomeric structure $[3,4]$, facilitating the cloning of the corresponding cDNA [5]. Our results establish that the membrane-bound enzyme is unique, both immunologically and structurally. This enzyme also has the unusual property of being activated as much as 20 -fold by thiol-reactive reagents [6]. Our studies on the amino acid sequence of microsomal glutathione transferase have shown that modification by thiol-reactive reagents occurs at the single cysteine residue, namely cysteine-49 [4].

Early on we noted that microsomal glutathione transferase can also be activated by limited proteolysis with trypsin [7]. The present study describes the determination of the cleavage sites correlated with trypsin activation, the effects of certain other proteinases, the properties of the activated enzyme, and the relationship between activation by limited proteolysis and by a thiol-reactive reagent. These studies indicate that activation is accompanied by a conformational change of the enzyme.

\section{MATERIALS AND METHODS}

Proteins

Microsomes and microsomal glutathione transferase were prepared from the livers of male Sprague-Dawley rats $(180-200 \mathrm{~g})$ as described [4]. Trypsin, lysine-specific proteinase (Boehringer, Mannheim, Germany), trypsin inhibitor and insoluble trypsin [tosylphenylalanylchloromethane ('TPCK')-treated] bound to agarose (Sigma Chemical Co., St. Louis, MO, U.S.A.) were from the sources indicated.

\section{Trypsin activation}

Aliquots of the purified enzyme $(0.3-0.5 \mathrm{mg} / \mathrm{ml})$ in $0.01 \mathrm{M}$-potassium phosphate $(\mathrm{pH} 8) / 0.1 \mathrm{M}-\mathrm{KCl} / 0.1 \mathrm{~mm}-$ EDTA $/ 1 \mathrm{~mm}-\mathrm{GSH} / 0.1-1 \%$ Triton X-100/20\% (v/v) glycerol were incubated with trypsin $(1 \mathrm{mg} / \mathrm{ml})$ at room temperature for the times indicated. Reactions were stopped by the addition of a 2-fold excess of trypsin inhibitor and by transfer of the sample to an ice bath. Other additions were made in certain cases as described. Incubations with lysine-specific proteinase were identical, except that half as much proteinase $(0.5 \mathrm{mg} / \mathrm{ml})$ was used.

In the case of rat liver microsomes, fresh preparations $(3 \mathrm{mg} / \mathrm{ml})$ were incubated with trypsin $(40 \mu \mathrm{g} / \mathrm{ml})$ in $0.07 \mathrm{M}$-potassium phosphate $(\mathrm{pH} 7.6) / 50 \mathrm{~mm}$-sucrose at $25^{\circ} \mathrm{C}$. Controls received either no trypsin or a 3-fold excess of trypsin inhibitor before the addition of trypsin.

\section{Glutathione transferase activity}

This was assayed with 1-chloro-2,4-dinitrobenzene as the second substrate as described in $[6,8]$.

\section{Activation with $\boldsymbol{N}$-ethylmaleimide ( $2 \mathrm{mM}$ )}

This was perforrined as described [6,9].

\section{SDS/polyacrylamide-ge! electrophoresis}

This was performed according to Laemmli [10], except 


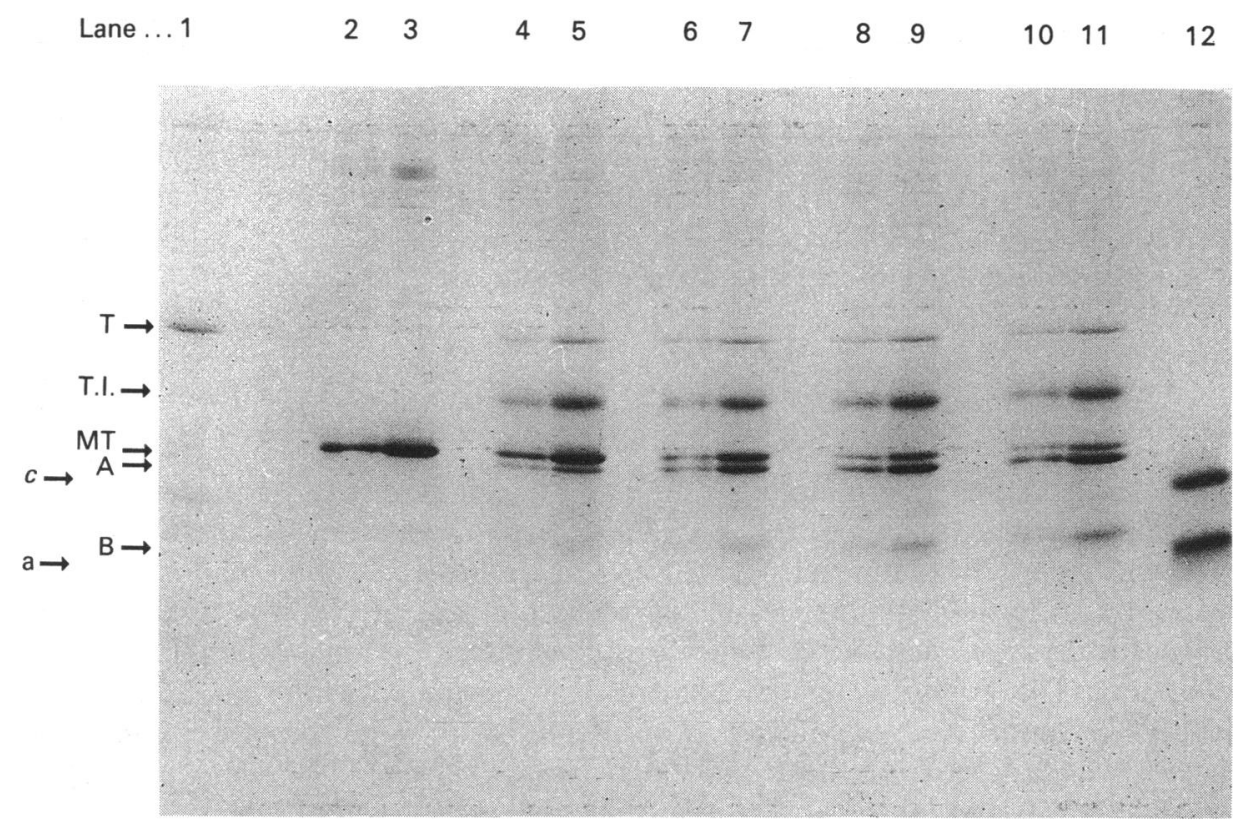

Fig. 1. SDS/polyacrylamide-gel electrophoresis showing the cleavage of microsomal glutathione transferase by trypsin

Trypsin treatment was performed as described in the Materials and methods section. Lane 1, trypsin (2 $\mu \mathrm{g})$. Lanes 2 and 3, 2 and $5 \mu \mathrm{g}$ of microsomal glutathione transferase respectively. Lanes 4 and 5,2 and $5 \mu \mathrm{g}$ of microsomal glutathione transferase respectively after $15 \mathrm{~min}$ of digestion at room temperature with trypsin $(0.25 \mathrm{mg} / \mathrm{ml})$ stopped with a 2 -fold excess of trypsin inhibitor. Lanes 6 and $7(30 \mathrm{~min}), 8$ and $9(45 \mathrm{~min}), 10$ and $11(60 \mathrm{~min})$ are as lanes 4 and 5 with longer incubation times as indicated. Lane 12 contains $2 \mu \mathrm{g}$ each of cytochrome $c(12.5 \mathrm{kDa})$ and aprotinin $(6.5 \mathrm{kDa})$ as $M_{\mathrm{r}}$ standards. Arrows indicate T (trypsin), T.I. (trypsin inhibitor), MT (microsomal glutathione transferase), A (fragment A), B (fragment B), $c$ (cytochrome $c$ ) and a (aprotinin).

that the Tris/ $\mathrm{HCl}$ concentration in the separation gel was doubled in order to improve resolution. The gels were stained with Coommassie Brilliant Blue R-250 and subsequently scanned with an LKB laser scanner with automatic integration.

\section{Amino acid sequence analysis}

Amino acid sequence analysis of the purified enzyme $(0.25 \mathrm{mg} / \mathrm{ml})$ after maximal activation with trypsin (trypsin bound to agarose; $50 \%$ packed gel in the same buffer as described above) was performed with a liquidphase sequencer after dialysis as described in [4]. Hydrazinolysis [11] was performed on aliquots containing $2.5 \mathrm{nmol}$ of native enzyme or enzyme activated with insoluble trypsin.

\section{Protein}

This was determined by the method of Peterson [12].

\section{RESULTS}

The gel-electrophoretic pattern observed with purified microsomal glutathione transferase treated with trypsin for different times is shown in Fig. 1. Two fragments were seen, and on some gels a third, smaller, fragment was also visible (Fig. 2a). The integrated areas for the different fragments as a percentage of the total are related to enzyme activity in Fig. 2(b). It is apparent that proteolysis of the purified enzyme by trypsin requires relatively large amounts of the proteinase and is a slow process in comparison with activation of the enzyme in situ in microsomes (Fig. 3), where much less trypsin was needed per $\mathrm{mg}$ of protein and the processes of both activation and denaturation were faster. As is the case for $N$ ethylmaleimide [6], trypsin does not activate cytosolic glutathione transferase (results not shown).

In order to determine the tryptic cleavage sites correlated with the activation process, the purified enzyme was digested to give maximal activation, using trypsin bound to agarose beads. The amino acid sequence of the enzyme was subsequently analysed by liquidphase-sequencer degradation. Two sequences starting after Lys-4 and Lys-41 were detected in approximately equal amounts (Table 1). The positions of the corresponding cleavage sites in the whole polypeptide chain are shown in Fig. 4. From these starting positions and apparent molecular masses the fragments observed on SDS/polyacrylamide-gel electrophoresis (Figs. 1, 2, 5 and 6) can therefore be identified as encompassing positions 5-154 (fragment A) and 42-154 (fragment B).

Lysine-specific proteinase also activates the enzyme and generates the same gel-electrophoretic pattern (except for an additional smaller fragment) (Fig. 5), demonstrating that the cleavages that correlate with activation follow lysine residues. Cleavage at the $C$-terminal region of the enzyme by trypsin or lysine-specific proteinase is not likely, because there is no lysine residue closer to the $C$-terminus than at position 67 [5]. The content of $C$-terminal amino acids was determined after activation with insoluble trypsin (Table 2). Although the 

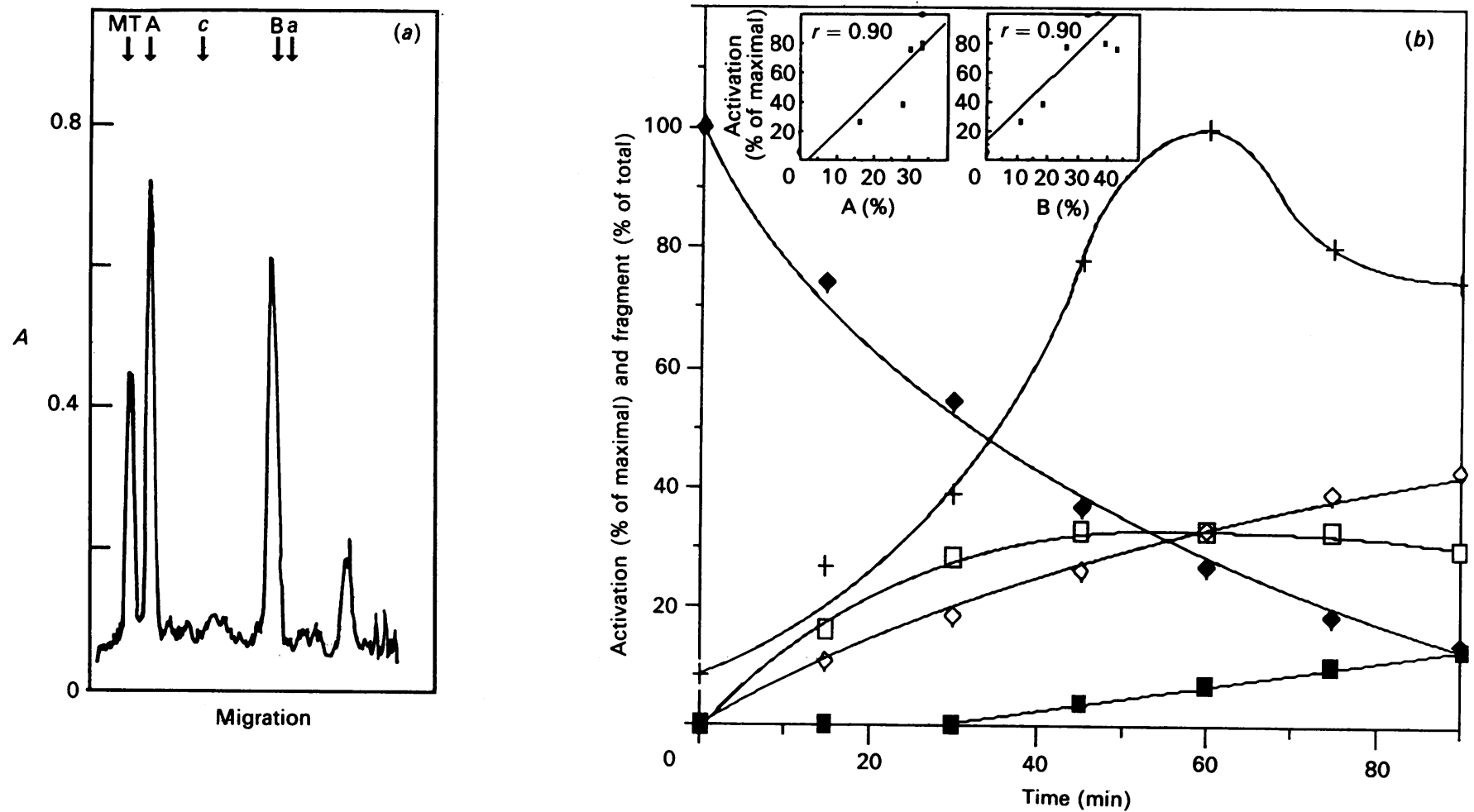

Fig. 2. Analysis of the activation of microsomal glutathione $S$-transferase after specific tryptic cleavage

(a) Gel scan of lane obtained after trypsin treatment for $75 \mathrm{~min}$. Positions of arrows indicate: MT (microsomal glutathione transferase), A (fragment A), B (fragment B), $c$ (cytochrome c) and a (aprotinin). [ $c=$ cytochrome $c(12.5 \mathrm{kDa})$ and a $=$ aprotinin $(6.5 \mathrm{kDa})$ ]. The lower part of the gel is shown. $(b)$ Relative amounts of uncleaved and cleaved microsomal glutathione transferase after different periods of trypsin treatment in relation to the degree of activation. + , Percentage of maximal activation; $\diamond$, relative amount of uncleaved enzyme; $\square$, fragment A (5-154); $\diamond$, fragment B (42-154); $\square$, a smaller fragment not visible in all experiments (as determined by integrated gel scans). The insets show the correlation between the percentage of fragment $\mathrm{A}$ or $\mathrm{B}$ generated and percentage of maximal activation.

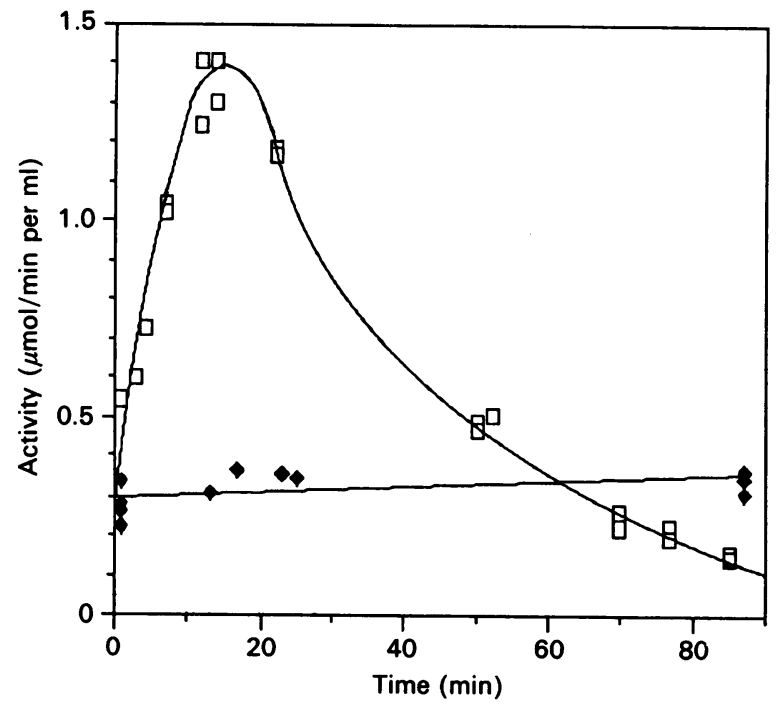

Fig. 3. Glutathione transferase activity in rat liver microsomes treated with trypsin for different periods of time

$\square$, Trypsin-treated sample; $\diamond$, control. Details are given in the Materials and methods section.

analysis was only semiquantitative, lysine and the expected leucine (Fig. 4) were detected, whereas only the latter was found in untreated controls.
Table 1. Liquid-phase sequencer determination of the $N$-terminal sequences of microsomal glutathione transferase maximally activated by proteolysis with trypsin as described in the Materials and methods section

\begin{tabular}{cl}
\hline Position & Amino acids \\
\hline 1 & Gln, Val \\
2 & Leu, Phe \\
3 & Met, Ala \\
4 & Asp, Asn \\
5 & Asn, Pro
\end{tabular}

Having established that limited proteolysis with trypsin cleaves the enzyme at two sites, the question arises as to which cleavage is involved in activation. In an attempt to answer this question, we included bromosulphophthalein (BSP), an inhibitor of microsomal glutathione transferase [13], during the trypsin treatment. This inhibitor proved to alter the relative amounts of fragments A and B obtained in a concentration-dependent manner, from $49 \% \mathrm{~A}-35 \% \mathrm{~B}$ in the absence of BSP to $15 \% \mathrm{~A}-79 \%$ $B$ with an optimal concentration of this inhibitor (Figs. $6 a$ and $6 b$ ). The degree of activation was significantly correlated only with the relative amount of fragment B (inset to Fig. 6b). However, the same analysis of the data for enzyme cleaved with trypsin in the absence of BSP 


\section{$\theta$ \\ f \\ ADLKQLMDNEVLMAFTSYATIILAKMMFLSSATAFQRLTNKVFANPEDC \\ AGFGKGENAKKFLRTDEKVERVRRAHLNDLENIVPFLGIGLLYSLSGPDL STALIHFRIFVGARIYHTIAYLTPLPQPNRGLAFFVGYGVTLSMAYRLLRS \\ RLYL}

Fig. 4. Positions of cleavage in the amino acid sequence of microsomal glutathione transferase after maximal activation by trypsin

The 'knives' indicate the sites of proteolytic cleavage at Lys-4 and Lys-41. The reactive Cys-49 residue is underlined.

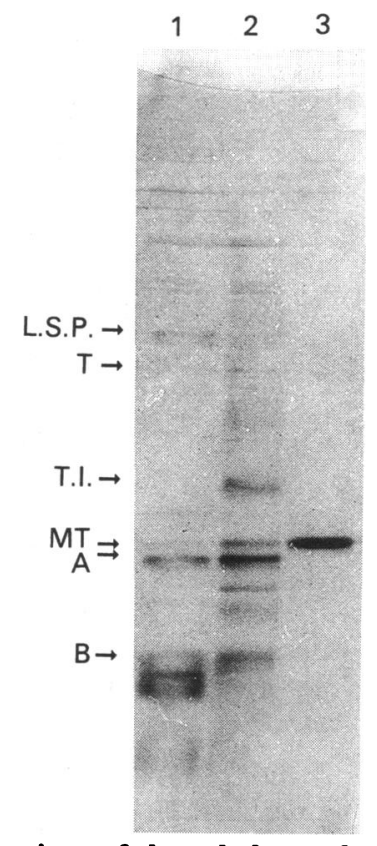

Fig. 5. Comparison of the gel-electrophoretic patterns obtained after activation of microsomal glutathione transferase with lysine-specific proteinase and with trypsin

Lane 1 is microsomal glutathione transferase $(2 \mu \mathrm{g})$ maximally activated by lysine-specific proteinase, and lane 2 is the same amount of enzyme activated with trypsin. Lane 3 is enzyme only. Arrows indicate L.S.P. (lysinespecific proteinase), T (trypsin), T.I. (trypsin inhibitor), MT (microsomal glutathione transferase), A (fragment A) and $B$ (fragment $B$ ).

yields a significant correlation of activity with the relative amounts of both fragments A and B (inset to Fig. 2).

Figs. 6(a) and 6(b) also show that activation of the enzyme with $N$-ethylmaleimide before trypsin treatment renders the Lys-41 bond more susceptible to proteolysis, and that the enzyme fully cleaved at this position retains maximal activity. In fact, the enzyme treated in this manner is more stable to the further degradation that occurs upon trypsin treatment for long time periods.

The effect of trypsin treatment on the kinetic parameters of the enzyme are shown in Table 3 in comparison with those obtained after activation with $N$-ethylmaleimide. The kinetic parameters change in a similar fashion after both types of activation. It therefore seems probable that both types of treatment cause similar changes in the microsomal glutathione transferase. This is likely to be a conformational change, judging from the different susceptibilities in the activated and unactivated enzyme of Lys-41 to trypsin cleavage.

\section{DISCUSSION}

In the present study we have shown that cleavages at sites Lys- 4 and Lys- 41 are those correlated with activation of microsomal glutathione transferase with trypsin. It appears that both sites contribute to the activation, but to what extent is not possible to determine from the present analysis. No other cleavage site for trypsin is detected under the conditions used. Longer incubation times, however, inactivate the enzyme, probably by additional proteolysis (Fig. 2). We have earlier noted that large portions of this enzyme are insensitive to trypsin, even under optimal conditions for cleavage of the denatured enzyme used to generate peptides for amino acid sequence analysis [4].

The enzyme both in situ in microsomes and in purified form is activated by trypsin, although the latter requires more proteinase and longer incubation times. This might reflect a protective effect of GSH, which is present in purified enzyme preparations to enhance their stability. It is also conceivable that activation of the activity in microsomes proceeds through some other mechanism (i.e. destruction of an inhibitor). However, observations from immunoblotting experiments reveal that activation is accompanied by cleavage of the enzyme in microsomes also (results not shown). The degree of activation is greater for the purified enzyme than for the microsomal activity. This is consistent with the fact that cytosolic glutathione transferase activity associated with microsomes increases the background activity in this preparation [16].

The activation of microsomal glutathione transferase by trypsin and $N$-ethylmaleimide seems to be highly similar for the following reasons: (1) the effects are not additive, i.e., trypsin treatment does not further activate the enzyme that has been maximally activated by $N$ ethylmaleimide; and (2) the kinetic parameters of the enzyme change in the same manner in both cases. We have also noted similar changes in the sensitivity of the enzyme, to certain inhibitors after activation with trypsin or $N$-ethylmaleimide (E. Mosialou \& R. Morgenstern, unpublished work).

It is clear that at least the $N$-ethylmaleimide-modified enzyme can retain full activity after total cleavage at Lys41. For the unmodified enzyme the situation is less clear, but the evidence favours the conclusion that both trypsin cleavage sites can bring about activation. The question arises whether the cleavages cause the smaller peptides generated to dissociate from the enzyme or whether they 

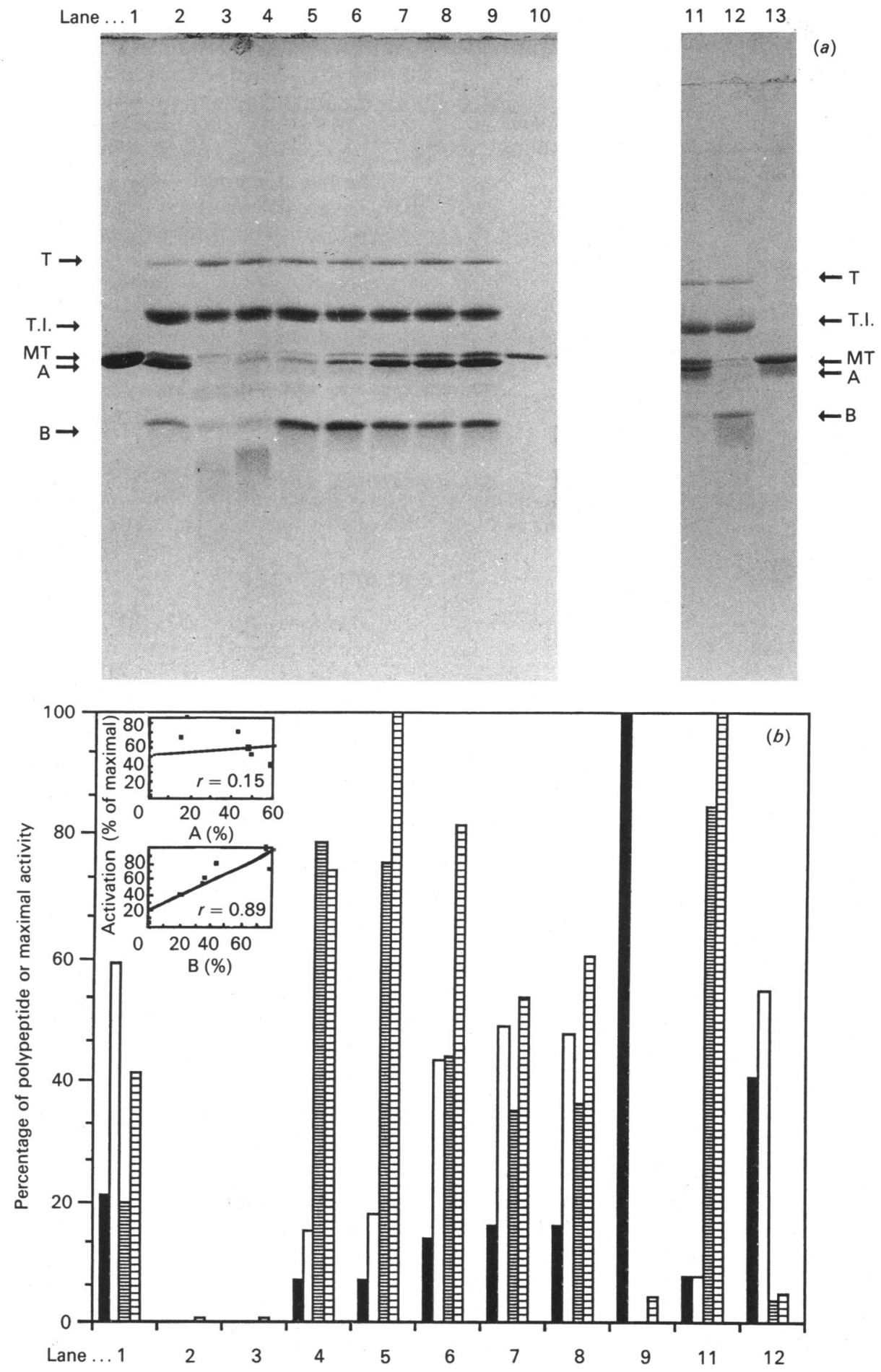

Fig. 6. Trypsin cleavage and activation either in the presence of BSP or after activation with $\boldsymbol{N}$-ethylmaleimide

(a) SDS/polyacrylamide-gel electrophoretograms of microsomal glutathione transferase treated with trypsin either in the presence of BSP or after $N$-ethylmaleimide activation. Lane 1 , microsomal glutathione transferase $(5 \mu \mathrm{g})$. Lane $2,5 \mu \mathrm{g}$ of enzyme treated with trypsin (see the Materials and methods section) for $120 \mathrm{~min}$. Lanes 3-9 are as lane 2, except that BSP was included during trypsin treatment at the following concentrations: $0.5,0.25,0.1,0.05,0.025,0.01$ and $0.001 \mathrm{~mm}$ respectively. Lane 10, as lane $1(1 \mu \mathrm{g})$. Lanes 11 and 12 , enzyme $(2 \mu \mathrm{g})$ treated with trypsin for 15 min (as in the Materials and methods section). The sample in lane 12 was activated with $2 \mathrm{~mm}-N$-ethylmaleimide before trypsin treatment [excess $N$-ethylmaleimide was conjugated by adding an equal amount of GSH (pH adjusted to 7) after maximal activation]. The control received GSH before $N$-ethylmaleimide and was therefore not activated, but had the same amounts of reagents present during trypsin treatment. Lane 13, as lane $1(2 \mu \mathrm{g})$. Arrows indicate T (trypsin), T.I. (trypsin inhibitor), MT (microsomal glutathione transferase), A (fragment A) and B (fragment B). (b) Bar chart illustrating the relative amounts of fragments after trypsin cleavage of microsomal glutathione transferase in relation to relative activation. Scans were run on the corresponding lanes in (a). $\square, \% \mathrm{MT} ; \square, \% \mathrm{~A}$; 圈, \% B; 目, \% of $V_{\max }$. The inset shows the relationship between percentage of maximal activation and percentage of fragment generated. 
Table 2. Recovery of $C$-terminal amino acids as determined by hydrazinolysis of trypsin-treated and untreated microsomal glutathione transferase

Details are given in the Materials and methods section. Glycine from GSH was also detectable. Portions $(2.5 \mathrm{nmol})$ of enzyme were subjected to hydrazinolysis; values are means of duplicates.

$C$-Terminal amino acids recovered $(\%)$

Amino acid Enzyme... Trypsin-treated Untreated

\begin{tabular}{lrc}
\hline Leucine & 56 & 28 \\
Lysine & 216 & -
\end{tabular}

Table 3. Kinetic parameters for microsomal glutathione transferase after maximal activation with trypsin or $N$ ethylmaleimide (NEM)

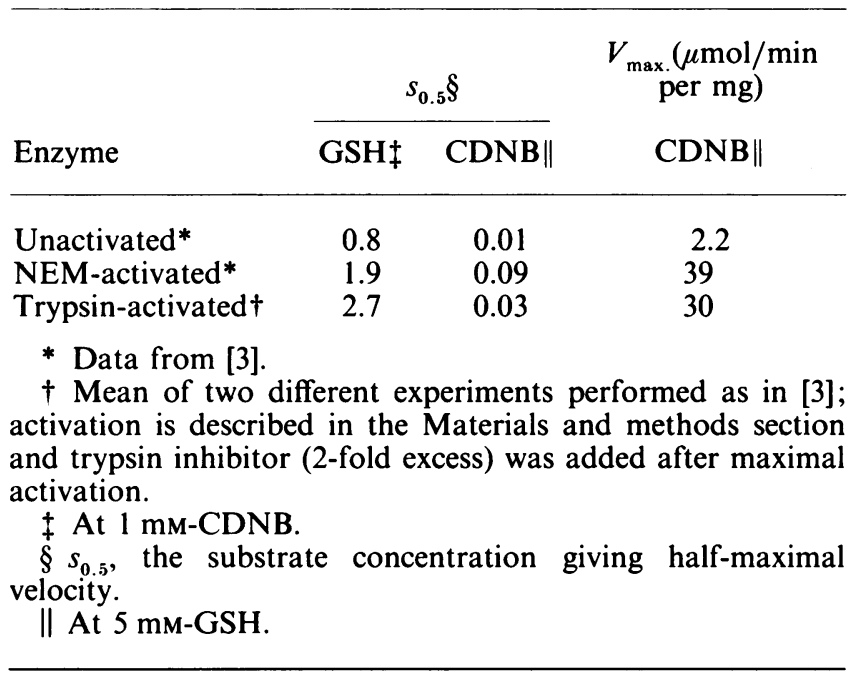

remain attached to the protein. This question will require further experiments on membrane topology. However, trypsin treatment does not cause release of the enzyme activity from microsomes. Since the membrane-anchor sequence is suspected to be positioned $N$-terminally of Lys-41, this would favour the idea that cleavage occurs without dissociation of the enzyme fragments. This type of enzyme modification involving both activation and proteolytic cleavage, also occurs with zymogens, e.g. chymotrypsinogen [17]. The fact that proteolysis and thiol-reactive-reagent modification at different sites can both bring about activation of microsomal glutathione transferase indicates that the precise position of modification is not of crucial importance to this process, and that at least part of the $N$-terminal region of the enzyme might not be involved in the catalytic process. Since the whole protein is small, the function resides in comparatively short polypeptide segments.

Whether the microsomal glutathione transferase can be activated by limited proteolysis in vivo is of great interest. It has been shown that the activity of the enzyme can be increased by treating whole animals with toxic substances such as carbon tetrachloride, dibromoethane [18] and phorone [19]. The time periods required are relatively short, i.e., too short to allow induction. Therefore the possibility of activation involving proteolysis as an 'in vivo' defence against exposure to xenobiotics requires further study.

These studies were supported by grants from the Swedish Cancer Society and the Swedish Natural and Medical Science Research Councils.

\section{REFERENCES}

1. Mannervik, B. (1985) Adv. Enzymol. Relat. Areas Mol. Biol. 57, 357-417

2. Chasseaud, L. F. (1979) Adv. Cancer Res. 29, 175-274

3. Morgenstern, R. \& DePierre, J. W. (1985) Rev. Biochem. Toxicol. 7, 67-104

4. DeJong, J. L., Morgenstern, R., Jörnvall, H., DePierre, J. W. \& Tu, C.-P. D. (1988) J. Biol. Chem. 263, 8430-8436

5. Morgenstern, R., DePierre, J. W. \& Jörnvall, H. (1985) J. Biol. Chem. 260, 13976-13983

6. Morgenstern, R. \& DePierre, J. W. (1983) Eur. J. Biochem. 134, 591-597

7. Morgenstern, R. (1980) Abstr. Linderström-Lang Conf. Conjugation Reactions Drug Biotransformation 10th, Stockholm, Sweden

8. Habig, W. H., Pabst, M. J. \& Jakoby, W. B. (1974) J. Biol. Chem. 249, 7130-7139

9. Morgenstern, R., DePierre, J. W. \& Ernster, L. (1979) Biochem. Biophys. Res. Commun. 87, 657-663

10. Laemmli, U. K. (1970) Nature (London) 227, 680-685

11. Schroeder, W. A. (1972) Methods Enzymol. 25, 138-142

12. Peterson, G. L. (1977) Anal. Biochem. 83, 346-356

13. Smith, G. J. \& Litwack, G. (1980) Rev. Biochem. Toxicol. 2, 1-47

14. Reference deleted

15. Reference deleted

16. Morgenstern, R., Guthenberg, C., Mannervik, B. \& DePierre, J. W. (1983) FEBS Lett. 160, 264-268

17. Blow, D. M. (1971) Enzymes 3rd Ed. 3, 185-212

18. Botti, B., Moslen, M. T., Trieff, N. M. \& Reynolds, E. S. (1982) Chem.-Biol. Interact. 42, 259-270

19. Masukawa, T. \& Iwata, H. (1986) Biochem. Pharmacol. $35,435-438$ 